\title{
Effect of Non-Routine Perioperative Laboratory Values on 30 Day Mortality after Non-Cardiac Surgery
}

\author{
Michael Ohebsion, Zhongjie Cai, Alexandra Ruan, Stephanie Biglarian, Ling Zheng, \\ Mary M. Joseph \\ Departments of Anesthesiology and Neurology, Keck School of Medicine, University of Southern California, Los Angeles, \\ California \\ Email: mohebsio@usc.edu
}

How to cite this paper: Ohebsion, M., Cai, Z.J., Ruan, A., Biglarian, S., Zheng, L. and Joseph, M.M. (2017) Effect of Non-Routine Perioperative Laboratory Values on 30 Day Mortality after Non-Cardiac Surgery. Open Journal of Anesthesiology, 7, 166-173. https://doi.org/10.4236/ojanes.2017.76018

Received: March 28, 2017

Accepted: June 25, 2017

Published: June 28, 2017

Copyright $\odot 2017$ by authors and Scientific Research Publishing Inc. This work is licensed under the Creative Commons Attribution International License (CC BY 4.0).

http://creativecommons.org/licenses/by/4.0/

\section{(c) (i) Open Access}

\begin{abstract}
Background: It is known that certain laboratory value abnormalities may increase mortality in patient populations with specific risk factors and specific procedures. The purpose of this paper was to investigate the effect of drawing a non-routine lab (regardless of its result) on 30 day mortality and to investigate the effect of an abnormal non-routine laboratory value on 30 day mortality. Methods: The electronic medical records of adult patients undergoing non-cardiac surgery from 2011-2014 at LAC + USC Medical Center were reviewed retrospectively as approved by our institutional review board. Demographic data, surgical risk assessment, and routine and non-routine laboratory values (TSH, Troponin, Creatine Kinase, $\mathrm{Hb}$ Alc) were recorded; patients were grouped according to the single non-routine lab they had drawn. Multivariate logistic regression analysis was used to determine significant risk factors associated with increased 30 day mortality. Results: 11,306 patients met our inclusion criteria. Drawing a non-routine lab was associated with a statistically significant increase in mortality in the Troponin group ( $\mathrm{OR}=3.05, P<$ 0.001 ) but not in the TSH, Creatine Kinase, or Hb Alc groups. An abnormal non-routine lab value was associated with a statistically significantly increase in mortality in the TSH group (OR $=11.07, P=0.008)$ but not in the other three groups. Conclusions: Drawing a non-routine lab was associated with a statistically significant increase in mortality in the Troponin group but not in the TSH, Creatine Kinase, or Hb Alc groups. An abnormal TSH value was associated with a statistically significant increase in mortality but not an abnormal Troponin, Creatine Kinase, or $\mathrm{Hb} \mathrm{Alc}$. Routine and non-routine laboratory testing may guide perioperative patient care and result in improved post-surgical outcome.
\end{abstract}




\section{Keywords}

Laboratory Values, Perioperative, Non-Routine, 30 Day Mortality

\section{Background}

The use of laboratory (lab) testing prior to surgery is important in determining a patient's eligibility for a procedure and risk for postoperative complications. It is known that certain lab value abnormalities may increase mortality in patient populations with specific risk factors or undergoing specific procedures. For example, Cecconi, et al. (2016) found that hypernatremia was associated with an increase in mortality in patients undergoing major surgery [1]. However, the efficacy of non-selective laboratory testing in certain patient populations, namely low risk, has been questioned [2] [3]. While is has been commonplace to receive preoperative "routine" laboratory testing (such as sodium, potassium, etc.), the usefulness of non-routine laboratory testing has yet to be investigated thoroughly. In 2012, the American Society of Anesthesiologists Task Force on Preanesthesia Evaluation stated upon review of the literature that selective preoperative tests (based on the history, physical, and plan) may aid in decision-making and patient management while routine preoperative tests fail to make a significant contribution [4]. The purpose of this paper was first, to investigate the effect of drawing a non-routine lab, regardless of its result, on 30 day mortality (denoted as the "Lab Drawing Analysis"), and second, to investigate the effect of an abnormal non-routine lab value on 30 day mortality (denoted as the "Abnormal Lab Analysis").

\section{Methods}

The electronic medical records of adult patients undergoing non-cardiac surgery from 2011-2014 at LAC + USC Medical Center were reviewed retrospectively as approved by our institutional review board. Demographic data (age, gender, ethnicity, and ASA physical status), surgical risk assessment, emergent status, anesthesia type, date of death (if applicable), routine laboratory values, and nonroutine lab values (TSH, Troponin, Creatine Kinase, Hb A1c) were recorded.

To reach the final population, several adjustments were made to the original dataset of 102,884 records (see flow chart in Figure 1 below). First, all observations with missing data were removed. This included patients who were missing identifying information and patients who were missing routine labs. The routine labs included Hemoglobin, Platelets, Calcium, Carbon Dioxide, Chloride, Creatinine, Glucose, Potassium, Sodium, BUN, and Magnesium. Although magnesium is not widely recognized as a routine lab, the incidence of drawing magnesium at the institution from which the data derived was very similar to the incidence of drawing routine labs. Next, residual cases with cardiac or nonsurgical procedures listed, observations with ASA 6 physical status, and records with age 


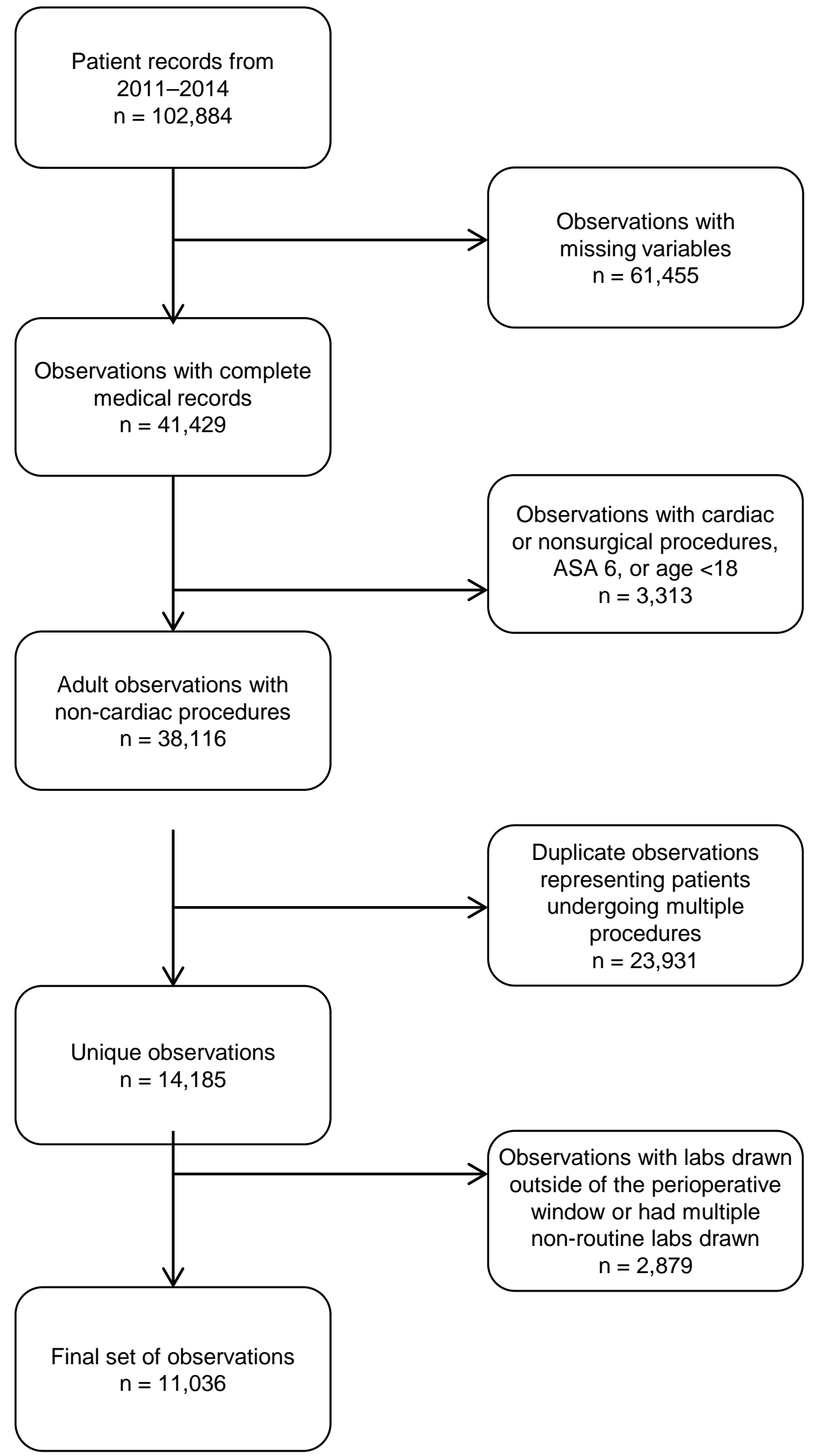




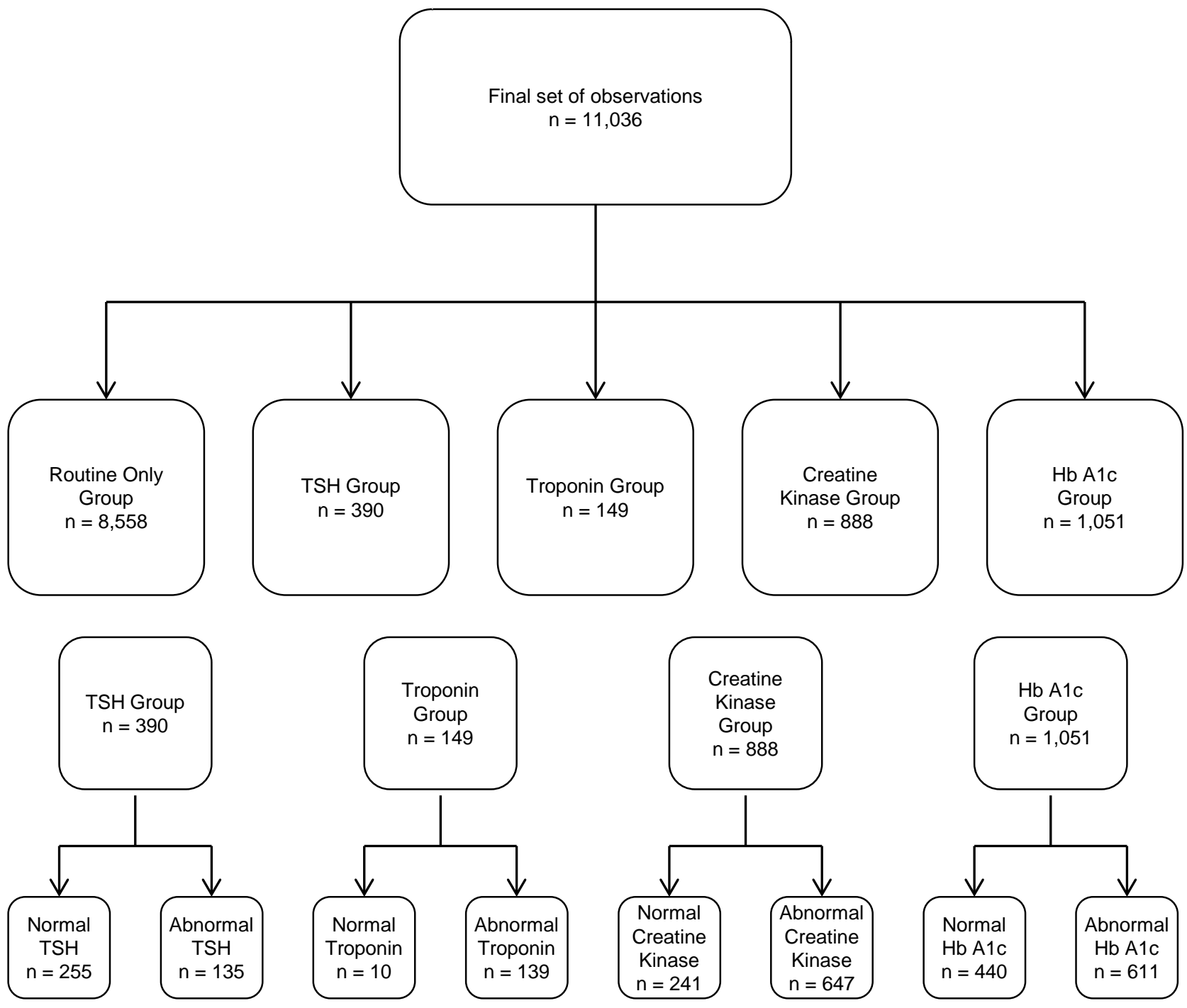

Figure 1. Flow chart.

less than 18 years were dropped. A surgical risk score (low, medium, high) was then assigned to each entry using the American College of Surgeons surgical risk classification [5]. Examples of high risk procedures included any intracranial surgery, liver transplants, and spinal reconstruction. Examples of medium risk procedures included renal transplants, cholecystectomies, and hip replacements. And examples of low risk procedures included breast biopsy, rhinoplasty, and cataract surgery.

Patients who underwent multiple procedures during one hospital stay had multiple entries in the data, each representing a different procedure they underwent. Therefore, duplicates were removed, leaving only the entry that represented the highest risk procedure the patient underwent. For example, a patient undergoing two low risk, three medium risk, and one high risk procedure all in one hospital stay had six separate entries in the original dataset; in that scenario the high risk procedure would remain while all others were dropped. Next, cases whose most recent labs were taken outside of the perioperative window, defined 
as 90 days prior to surgery to 30 days post-surgery, were excluded. Lastly, cases that had multiple non-routine labs were excluded in order to reduce confounding effects and isolate the effect of a single non-routine lab on mortality. After making the above adjustments, 11,036 cases made up the final population.

For the Lab Drawing Analysis, the final population was split into five separate groups based on which one (or none) of the four non-routine labs the patient had drawn: routine labs only, routine labs plus TSH (the "TSH group"), routine labs plus Troponin, routine labs plus Creatine Kinase, and routine labs plus $\mathrm{Hb}$ A1c. Because one of the criteria was to remove observations with more than one non-routine labs drawn, no patient fell into more than one of the above groups.

For the Abnormal Lab Analysis, each of the four non-routine lab groups from the Lab Drawing Analysis was divided into those with a normal non-routine lab result and those with an abnormal non-routine lab result. Abnormally high values were not distinguished from abnormally low values.

The incidence of 30 day mortality in each of the groups was calculated. Multivariate logistic regression analysis was used to control for age, gender, ethnicity, surgical risk stratification, ASA physical status, and emergent status. The Routine Only group was used as the control for each non-routine lab groups in the Lab Drawing Analysis. In the Abnormal Lab Analysis, cases with normal non-routine labs were used as controls against their abnormal non-routine lab counterparts.

\section{Results}

\subsection{Lab Drawing Analysis}

Figure 2 compares the incidence of mortality in patients with only routine labs drawn to those who had routine labs plus one of the non-routine labs recorded (divided into elective, emergent, and combined elective plus emergent cases). The mortality in the Routine Only group was $1.85 \%$. Each of the non-routine lab groups had a higher mortality, including $4.36 \%$ in the TSH group and $18.12 \%$ in the Troponin group.

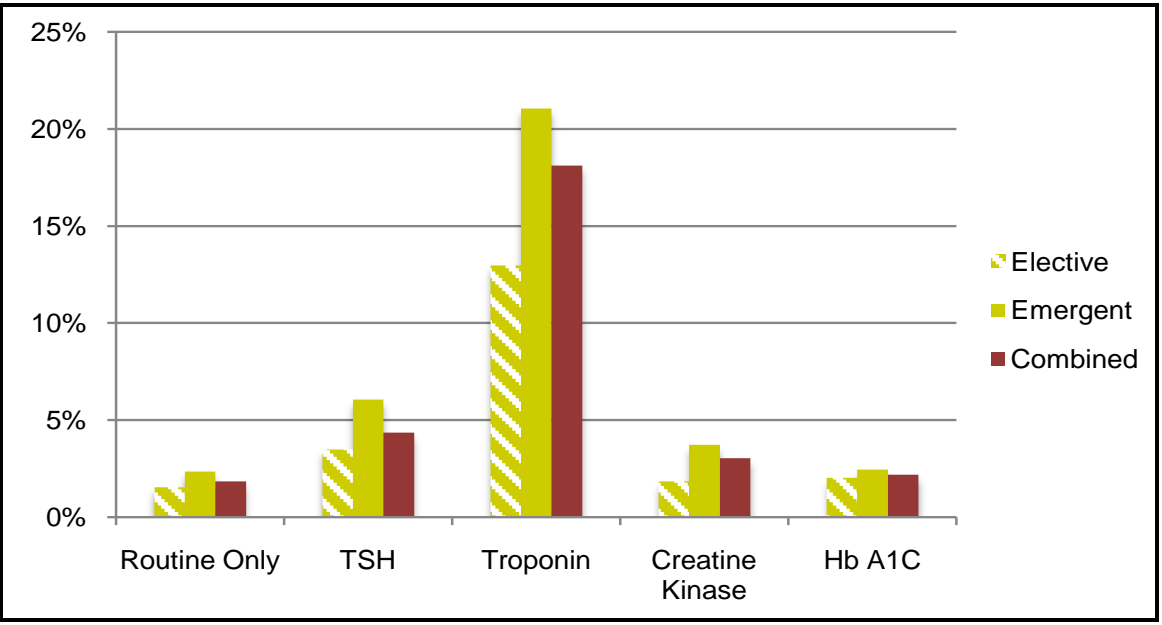

Figure 2. Incidence of mortality, routine versus non-routine groups. 
Table 1 contains the results of a multivariate analysis comparing the incidence of mortality in the Routine Only group to each of the non-routine groups. Drawing a non-routine lab was associated with a statistically significant increase in mortality in the Troponin group ( $\mathrm{OR}=3.05, P<0.001$ ). However, drawing $\mathrm{TSH}$, Creatine Kinase, or $\mathrm{Hb}$ Alc was not associated with a statistically significant increase in mortality.

\subsection{Abnormal Lab Analysis}

Figure 3 compares the incidence of mortality in the normal non-routine groups to that in the abnormal non-routine groups (divided into elective, emergent, and combined elective plus emergent cases). Mortality was higher in the abnormal TSH and abnormal Troponin groups than the normal TSH and Troponin groups, respectively. For TSH, mortality was $3.14 \%$ in the normal group versus $6.67 \%$ in the abnormal group. For Troponin, mortality was $10.00 \%$ in the normal group versus $18.71 \%$ in the abnormal group.

Table 2 contains the results of a multivariate analysis comparing the incidence of mortality in each of the normal non-routine lab groups to the abnormal non-routine lab groups. An abnormal TSH value was associated with a statistically significant increase in mortality $(\mathrm{OR}=11.07, P=0.008)$. However, an abnormal Troponin, Creatine Kinase, or Hb Alc was not associated with a statistically significant increase in mortality.

Table 1. Association of drawing non-routine lab with 30 day mortality.

\begin{tabular}{cccccc}
\hline Non-Routine Lab & Sample Size & OR & \multicolumn{2}{c}{$95 \%$ CI } & P-Value \\
\hline TSH & 8948 & 1.24 & 0.70 & 2.20 & 0.466 \\
Troponin & 8707 & 3.05 & 1.77 & 5.26 & $<0.001$ \\
Creatine Kinase & 9446 & 0.90 & 0.54 & 1.51 & 0.702 \\
Hb Alc & 9609 & 0.35 & 0.21 & 0.58 & $<0.001$ \\
\hline
\end{tabular}

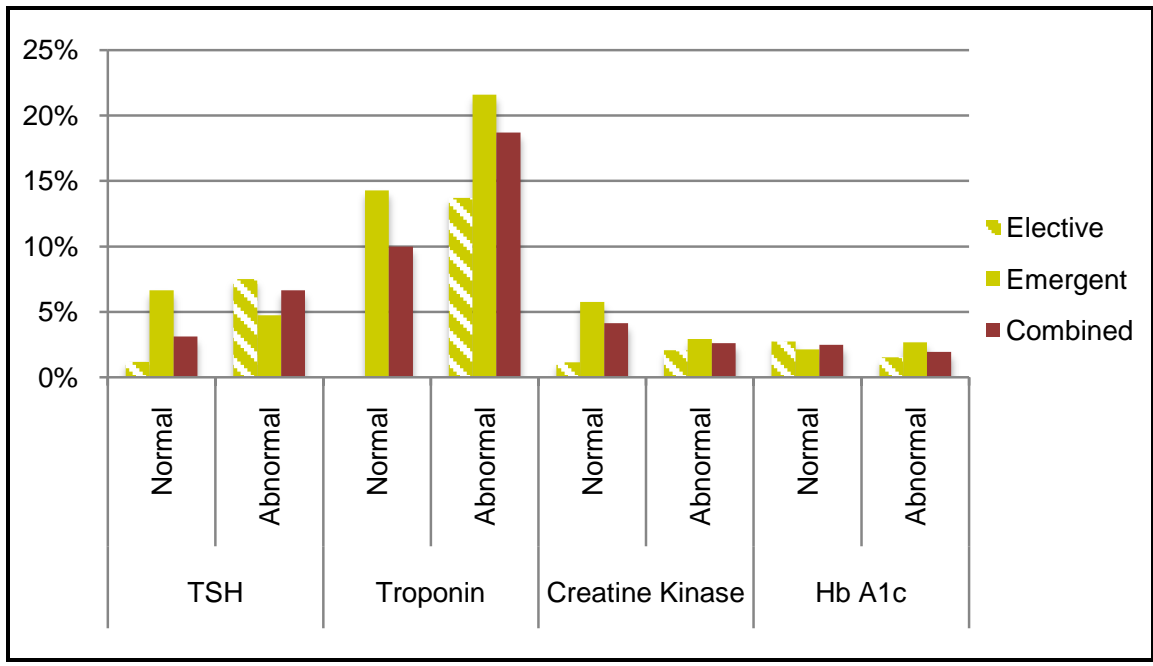

Figure 3. Incidence of mortality, normal versus abnormal non-routine groups. 
Table 2. Association of abnormal non-routine lab with 30 day mortality.

\begin{tabular}{cccccc}
\hline Non-Routine Lab & Sample Size & OR & \multicolumn{2}{c}{$95 \%$ CI } & P-Value \\
\hline TSH & 390 & 11.07 & 1.87 & 65.62 & 0.008 \\
Troponin & 149 & 0.63 & 0.05 & 8.22 & 0.724 \\
Creatine Kinase & 888 & 1.47 & 0.46 & 4.76 & 0.517 \\
Hb A1c & 1051 & 0.42 & 0.15 & 1.13 & 0.085 \\
\hline
\end{tabular}

\section{Discussion}

Drawing a non-routine lab was associated with a statistically significant increase in mortality in the Troponin group but not in the TSH, Creatine Kinase, or $\mathrm{Hb}$ Alc groups. Additionally, an abnormal TSH value was associated with a statistically significant increase in mortality but not an abnormal Troponin, Creatine Kinase, or $\mathrm{Hb} \mathrm{A1c}$, though clinical significance cannot be ruled out based on these results alone. The relatively smaller sample sizes in this study may contribute to the lack of statistically significant associations. For example, although a statistically significant result was not observed in the Troponin Group with a sample size of 149, Devereaux, et al. (2012) found a statistically significant association between a peak fourth-generation Troponin $\mathrm{T}$ value of at least $0.02 \mathrm{ng} / \mathrm{mL}$ and 30 day mortality in a study of 15,133 patients undergoing noncardiac surgery [6].

One of the limitations this study faced centered on the reporting of death date. The date of death was only recorded in patients who passed during an admission at LAC + USC. The death dates of those who passed away at home or at another institution were not acquired. It is understood that the effect of these omissions is to underestimate the incidence of mortality in all groups. However, the unique effect on each subgroup is unclear. Additionally, the decision to order labs was at the discretion of the presiding resident or attending. There was no universal protocol that dictated which labs to order. Finally, while the defined perioperative window may be suitable for certain labs such as $\mathrm{Hb} \mathrm{Alc}$, it may be less appropriate for others.

In order to further explore the role of perioperative laboratory testing and mortality risk, a prospective study may be beneficial. Future studies may benefit from exploring the prognostic differences between abnormally high and abnormally low laboratory values. Although the differentiation does not carry clinical significance for Creatine Kinase, Troponin, or $\mathrm{Hb} \mathrm{Alc}$, it will be clinically relevant for TSH. Ling, et al. (2013) found that overt hyperthyroidism was associated with an increased risk of complications in elderly patients following surgery for hip fractures [7]. Lastly, future studies may benefit from including additional identifying information such as co-morbidities and BMI as this data was not included due to reporting inconsistencies. Selective non-routine lab testing may guide perioperative patient care and result in improved post-surgical outcome.

\section{References}

[1] Cecconi, M., Hochrieser, H., Chew, M., Grocott, M., Hoeft, A., et al. (2016) Preo- 
perative Abnormalities in Serum Sodium Concentrations Are Associated with Higher In-Hospital Mortality in Patients Undergoing Major Surgery. British Journal of Anaesthesia, 116, 63-69. https://doi.org/10.1093/bja/aev373

[2] Kumar, A. and Srivastava, U. (2011) Role of Routine Laboratory Investigations in Preoperative Evaluation. Journal of Anaesthesiology, Clinical Pharmacology, 27, 174-179. https://doi.org/10.4103/0970-9185.81824

[3] Onuoha, O.C., Hatch, M.B., Miano, T.A. and Fleisher, L.A. (2015) The Incidence of Un-Indicated Preoperative Testing in a Tertiary Academic Ambulatory Center, a Retrospective Cohort Study. Perioperative Medicine, 4, 4-14. https://doi.org/10.1186/s13741-015-0023-y

[4] Apfelbaum, J., Connis, R., Nickinovich, D., Pasternak, L., Arens, J., et al. (2012) Practice Advisory for Preanesthesia Evaluation, an Updated Report by the American Society of Anesthesiologists Task Force on Preanesthesia Evaluation. Anesthesiology, 116, 522-538. https://doi.org/10.1097/ALN.0b013e31823c1067

[5] Fleisher, L.A., Fleischmann, K.E., Auerbach, A.D., Barnason, S.A., Beckman, J.A., et al. (2015) 2014 ACC/AHA Guideline on Perioperative Cardiovascular Evaluation and Management of Patients Undergoing Noncardiac Surgery: A Report of the American College of Cardiology/American Heart Association Task Force on Practice Guidelines. Journal of Nuclear Cardiology, 22, 162-215.

https://doi.org/10.1007/s12350-014-0025-z

[6] Devereaux, P.J., Chan, M.T., Alonso-Coello, P., Walsh, M., Berwanger, O., et al. (2012) Association between Postoperative Troponin Levels and 30-Day Mortality among Patients Undergoing Noncardiac Surgery. JAMA, 307, 2295-2304.

[7] Ling, X.W., Howe, T.S., Koh, J.S.B., Wong, M.K. and Ng, A.C. (2013) Preoperative Thyroid Dysfunction Predicts 30-Day Postoperative Complications in Elderly Patients with Hip Fracture. Geriatric Orthopaedic Surgery \& Rehabilitation, 4, 43-49. https://doi.org/10.1177/2151458513504215

\section{Submit or recommend next manuscript to SCIRP and we will provide best} service for you:

Accepting pre-submission inquiries through Email, Facebook, LinkedIn, Twitter, etc. A wide selection of journals (inclusive of 9 subjects, more than 200 journals)

Providing 24-hour high-quality service

User-friendly online submission system

Fair and swift peer-review system

Efficient typesetting and proofreading procedure

Display of the result of downloads and visits, as well as the number of cited articles

Maximum dissemination of your research work

Submit your manuscript at: http://papersubmission.scirp.org/

Or contact ojanes@scirp.org 